Bull. Austral. Math. Soc.

26B35, 26D10

VoL. $61(2000)$ [153-159]

\title{
ON AN INEQUALITY OF KOLMOGOROV AND STEIN
}

\author{
Ha Huy Bang and hoang Mai Le
}

A.N. Kolmogorov showed that, if $f, f^{\prime}, \ldots, f^{(n)}$ are bounded continuous functions on $\mathbb{R}$, then $\left\|f^{(k)}\right\|_{\infty} \leqslant C_{k, n}\|f\|_{\infty}^{1-k / n}\left\|f^{(n)}\right\|_{\infty}^{k / n}$ when $0<k<n$. This result was

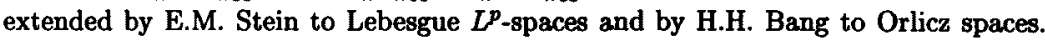
In this paper, the inequality is extended to more general function spaces.

\section{INTRODUCTION}

Kolmogorov [8] showed that, if $f, f^{\prime}, \ldots, f^{(n)}$ are bounded continuous functions on $\mathbf{R}$, then

$$
\left\|f^{(k)}\right\|_{\infty} \leqslant C_{k, n}\|f\|_{\infty}^{1-k / n}\left\|f^{(n)}\right\|_{\infty}^{k / n}
$$

when $0<k<n$, where $C_{k, n}=K_{n-k} / K_{n}^{1-k / n}$, and

$$
K_{i}=\frac{4}{\pi} \sum_{j=0}^{\infty} \frac{(-1)^{j(i+1)}}{(2 j+1)^{i+1}} .
$$

This is the best constant. Kolmogorov's result was extended to Lebesgue $L^{p}$-spaces by Stein [10] and to Orlicz spaces by Bang [1].

In this paper, the methods of these authors are modified to prove the analogous result for other function spaces on $\mathbb{R}$. For variants and applications of such results, see, for example, $[4,9,11]$. In particular, our results apply to amalgams of $L^{p}$ and $\ell^{q}$, as defined and studied in, for example, $[2,3,5,6,7]$.

To formulate our result, we need several definitions. First, if $f$ is a function on $\mathbf{R}$, we denote by $\tau(t) f$ its translate: $\tau(t) f(s)=f(s+t)$. Next, let $D(\mathbb{R})$ be a space of test functions, such as $C_{c}^{\infty}(\mathbb{R})$ or $\mathcal{S}(\mathbb{R})$. We require that translations act continuously in $D(\mathbb{R})$.

\section{Received 1st April, 1999}

Research supported by the National Basic Research Program in Natural Science.

The authors would like to express their sincere thanks to the Editor, Professor Michael G. Cowling, who has written the new version of this paper.

Copyright Clearance Centre, Inc. Serial-fee code: 0004-9727/00 \$A2.00+0.00. 
Let $X(\mathbb{R})$ be a Banach space of functions on $\mathbf{R}$. We say that $X(\mathbb{R})$ is $\tau$-stable provided that $\tau(t) f$ is in $X(\mathbb{R})$ whenever $t$ is in $\mathbb{R}$ and $f$ is in $X(\mathbb{R})$ and further there is a constant $C_{X}$ such that

$$
\|\tau(t) f\|_{X} \leqslant C_{X}\|f\|_{X} \quad \forall f \in X(\mathbb{R}) \quad \forall t \in \mathbb{R} .
$$

Examples of $\tau$-stable spaces include Lebesgue spaces, Lorentz spaces, Orlicz spaces, and, more generally, rearrangement invariant function spaces, and spaces involving derivatives, such as the Sobolev spaces $W^{p, k}(\mathbb{R})$. For all these spaces, $C_{X}=1$. Other examples of $\tau$-stable spaces include amalgams and weighted Lebesgue spaces $L^{p}(\mathbb{R}, w)$ of functions $f$ such that

$$
\left(\int_{\mathbf{R}}|f(t)|^{p} w(t) d t\right)^{1 / p}<\infty
$$

where the weight $w$ is positive, bounded, and bounded away from 0 . For these spaces, $C_{X}>1$ in general.

Let $\|f\|_{X}$ denote the norm of $f$ in $X(\mathbb{R})$, and for $g$ in $D(\mathbb{R})$, let $\|g\|_{X}$. denote the norm of duality with $X(\mathbb{R})$, that is,

$$
\|g\|_{X^{*}}=\sup \left\{\left|\int_{\mathbb{R}} g(t) f(t) d t\right|: f \in X(\mathbb{R}),\|f\|_{X} \leqslant 1\right\} .
$$

We say that $X(\mathbb{R})$ is $D(\mathbb{R})$-full if the map from $D(\mathbb{R})$ to $X(\mathbb{R})^{*}$ is continuous, so $\|g\|_{X^{*}}<$ $\infty$ for all $g$ in $D(\mathbb{R})$, and, if $f$ is in $D(\mathbb{R})^{*}$ (the dual space of $D(\mathbb{R})$ ) and

$$
\sup \left\{\left|\int_{\mathbf{R}} g(t) f(t) d t\right|: g \in D(\mathbb{R}),\|g\|_{X^{*}} \leqslant 1\right\}<\infty
$$

then $f$ is in $X(\mathbb{R})$ and $\|f\|_{X}$ is equal to the left hand side of the inequality above. For example, take $D(\mathbb{R})$ to be $C_{c}^{\infty}(\mathbb{R})$. If $X(\mathbb{R})$ is an amalgam of $L^{p}$ and $\ell^{p}$ where $1<p, q \leqslant \infty$ (in particular if $X(\mathbb{R})=L^{p}(\mathbb{R})$ for such $p$ ) then $X(\mathbb{R})$ is $D(\mathbb{R})$-full; however, if $X(\mathbb{R})=L^{1}(\mathbb{R})$, then $X(\mathbb{R})$ is not $D(\mathbb{R})$-full: the problem is the measures.

THEDREM 1. Suppose that $X(\mathbf{R})$ is a $\tau$-stable $D(\mathbf{R})$-full Banach space of functions on $\mathbb{R}$. [The word "functions" here is intended to include generalised functions such as distributions.] If $f$ and its generalised derivative $f^{(n)}$ are in $X(\mathbb{R})$, then $f^{(k)}$ is in $X(\mathbb{R})$ when $0<k<n$ and

$$
\left\|f^{(k)}\right\|_{X} \leqslant C_{X} C_{k, n}\|f\|_{X}^{1-k / n}\left\|f^{(n)}\right\|_{X}^{k / n}
$$

Proof: Take a function $h$ in $D(\mathbb{R})$, and define $F: \mathbb{R} \rightarrow \mathbb{C}$ by the formula

$$
F(t)=\int_{\mathbf{R}} f(s+t) h(s) d s=\int_{\mathbf{R}} f(s) h(s-t) d s .
$$


Now

$$
\begin{aligned}
\left|F(t)-F\left(t^{\prime}\right)\right| & =\left|\int_{\mathbf{R}} f(s)\left[h(s-t)-h\left(s-t^{\prime}\right)\right] d s\right| \\
& \leqslant\|f\|_{X}\left\|\tau(-t) h-\tau\left(-t^{\prime}\right) h\right\|_{X^{*}} \longrightarrow 0
\end{aligned}
$$

as $t \rightarrow t^{\prime}$ in $\mathbb{R}$, since $\tau(-t) h \rightarrow \tau\left(-t^{\prime}\right) h$ in $D(\mathbf{R})$ and hence in $X^{*}(\mathbf{R})$. Furthermore, $F$ is bounded, since

$$
|F(t)| \leqslant\|\tau(t) f\|_{X}\|h\|_{X^{*}} \leqslant C_{X}\|f\|_{X}\|h\|_{X^{*}}
$$

Moreover,

$$
F^{(n)}(t)=\int_{\mathbf{R}} f^{(n)}(t+s) h(s) d s,
$$

and so, similarly, $F^{(n)}$ is continuous and bounded, and

$$
\left\|F^{(n)}\right\|_{\infty} \leqslant C_{X}\left\|f^{(n)}\right\|_{X}\|h\|_{X^{*}}
$$

Finally, since $h^{(k)}$ is in $D(\mathbb{R})$ and

$$
F^{(k)}(t)=(-1)^{k} \int_{\mathbf{R}} f(s) h^{(k)}(s-t) d s,
$$

$F^{(k)}$ is also bounded and continuous.

By Kolmogorov's inequality applied to $F$,

$$
\begin{aligned}
\left\|F^{(k)}\right\|_{\infty} & \leqslant C_{k, n}\|F\|_{\infty}^{1-k / n}\left\|F^{(n)}\right\|_{\infty}^{k / n} \\
& \leqslant C_{X} C_{k, n}\|f\|_{X}^{1-k / n}\left\|f^{(n)}\right\|_{X}^{k / n}\|h\|_{X^{*}}
\end{aligned}
$$

Since $X(\mathbb{R})$ is $D(\mathbb{R})$-full, by hypothesis, and

$$
\left|\int_{\mathbf{R}} h(s) f^{(k)}(s) d s\right| \leqslant C_{X} C_{k, n}\|f\|_{X}^{1-k / n}\left\|f^{(n)}\right\|_{X}^{k / n}\|h\|_{X} .
$$

for all $h$ in $D(\mathbb{R})$, it follows that $f^{(k)}$ is in $X(\mathbb{R})$, and

$$
\left\|f^{(k)}\right\|_{X} \leqslant C_{X} C_{k, n}\|f\|_{X}^{1-k / n}\left\|f^{(n)}\right\|_{X}^{k / n}
$$

as required.

This theorem implies, for instance, Stein's theorem, except for $L^{1}(\mathbb{R})$. Similarly, it does not give a result for amalgams involving $L^{1}$. However, we have several extensions of this result which take care of these examples.

Let $X_{u}(\mathbf{R})$ denote the closed subspace of $X(\mathbb{R})$ of all functions $f$ such that

$$
\|\tau(t) f-f\|_{X} \rightarrow 0 \text { as } t \rightarrow 0
$$


CoRollary 2. Suppose that $X(\mathbb{R})$ is a $\tau$-stable $D(\mathbb{R})$-full Banach space of functions on $\mathbb{R}$. If $f$ is in $X_{u}(\mathbb{R})$ and its generalised derivative $f^{(n)}$ is in $X(\mathbb{R})$, then $f^{(k)}$ is in $X_{u}(\mathbb{R})$ when $0<k<n$.

ProOF: By the theorem,

$$
\begin{aligned}
\left\|\tau(t) f^{(k)}-f^{(k)}\right\|_{X} & \leqslant C_{X} C_{k, n}\|\tau(t) f-f\|_{X}^{1-k / n}\left\|\tau(t) f^{(n)}-f^{(n)}\right\|_{X}^{k / n} \\
& \leqslant C_{X} C_{k, n}\|\tau(t) f-f\|_{X}^{1-k / n}\left[C_{X}\left\|f^{(n)}\right\|_{X}+\left\|f^{(n)}\right\|_{X}\right]^{k / n} \\
& \longrightarrow 0
\end{aligned}
$$

as $t \rightarrow 0$, so $f^{(k)}$ is in $X_{u}(\mathbb{R})$, as required.

The second variant of the result involves another subspace of $X(\mathbb{R})$. Given a nonnegative integer $m$, we say that a Banach space $X(\mathbb{R})$ of functions on $\mathbb{R}$ is stable under multiplication by $C^{m}(\mathbb{R})$ if, whenever $f$ is in $X(\mathbb{R})$ and $\varphi, \varphi^{\prime}, \ldots, \varphi^{(m)}$ are bounded and continuous on $\mathbb{R}$, the pointwise product $\varphi f$ is in $X(\mathbb{R})$ and

$$
\|\varphi f\|_{X} \leqslant C_{X, m}\|f\|_{X}\|\varphi\|_{C^{m}}=C_{X, m}\|f\|_{X} \sum_{j=0}^{m}\left\|\varphi^{(j)}\right\|_{\infty} .
$$

If $X(\mathbb{R})$ is stable under multiplication by $C^{m}(\mathbb{R})$ for some $m$, then we denote by $X_{0}(\mathbb{R})$ the closed subspace of $X(\mathbb{R})$ of all functions $f$ for which

$$
\lim _{\varepsilon \rightarrow 0+}\left\|\varphi_{\varepsilon} f-f\right\|_{X}=0
$$

where $\varphi_{\varepsilon}(x)=e^{-\varepsilon x^{2}}$.

Corollary 3. Suppose that $X(\mathbf{R})$ is a $\tau$-stable $D(\mathbb{R})$-full Banach space of functions on $\mathbb{R}$, stable under multiplication by $C^{m}(\mathbb{R})$ for some nonnegative integer $m$. If $f$ is in $X_{0}(\mathbb{R})$ and its generalised derivative $f^{(n)}$ is in $X(\mathbb{R})$, then $f^{(k)}$ is in $X_{0}(\mathbb{R})$ when $0<k<n$.

Proof: By the theorem, $f^{(j)}$ is in $X(\mathbb{R})$, when $0 \leqslant j \leqslant n$. By Leibniz's rule for the derivative of a product,

$$
\begin{aligned}
\left\|\varphi_{\varepsilon} f^{(k)}-f^{(k)}\right\|_{X} & =\left\|\left(\varphi_{\varepsilon} f\right)^{(k)}-f^{(k)}-\sum_{j=0}^{k-1}\left(\begin{array}{l}
k \\
j
\end{array}\right) \varphi_{\varepsilon}^{(k-j)} f^{(j)}\right\|_{X} \\
& \leqslant\left\|\left(\varphi_{\varepsilon} f-f\right)^{(k)}\right\|_{X}+\sum_{j=0}^{k-1}\left(\begin{array}{l}
k \\
j
\end{array}\right)\left\|\varphi_{\varepsilon}^{(k-j)} f^{(j)}\right\|_{X^{*}}
\end{aligned}
$$

By the theorem,

$$
\left\|\left(\varphi_{\varepsilon} f-f\right)^{(k)}\right\|_{X} \leqslant C_{X} C_{k, n}\left\|\varphi_{\varepsilon} f-f\right\|_{X}^{1-k / n}\left\|\left(\varphi_{\varepsilon} f-f\right)^{(n)}\right\|_{X}^{k / n} \rightarrow 0
$$


as $\varepsilon \rightarrow 0$, since $\left\|\varphi_{\varepsilon} f-f\right\|_{X} \rightarrow 0$ and $\left\|\left(\varphi_{\varepsilon} f-f\right)^{(n)}\right\|_{X}$ is bounded, by another application of Leibniz's rule. Further, when $0 \leqslant j<k$,

$$
\left\|\varphi_{\varepsilon}^{(k-j)} f^{(j)}\right\|_{X} \leqslant C_{X, m}\left\|\varphi_{\varepsilon}^{(k-j)}\right\|_{C^{m}}\left\|f^{(j)}\right\|_{X}
$$

and it is easy to check that $\left\|\varphi_{\varepsilon}^{(k-j)}\right\|_{C^{m}} \rightarrow 0$ as $\varepsilon \rightarrow 0^{+}$.

A third variant of the result combines the themes of the two previous corollaries. Assume that $X(\mathbb{R})$ is stable under multiplication by $C^{m}(\mathbb{R})$, and let $X_{1}(\mathbb{R})$ denote the subspace of $X(\mathbb{R})$ of all functions $f$ such that $\varphi_{\varepsilon} f$ is in $X_{u}(\mathbb{R})$ for all $\varepsilon$ in $\mathbb{R}^{+}$.

COROLlaRY 4. Suppose that $X(\mathbb{R})$ is a $\tau$-stable $D(\mathbb{R})$-full Banach space of functions on $\mathbb{R}$, stable under multiplication by $C^{m}(\mathbb{R})$ for some integer $m$. If $f$ is in $X_{1}(\mathbf{R})$ and its generalised derivative $f^{(n)}$ is in $X(\mathbb{R})$, then $f^{(k)}$ is in $X_{1}(\mathbb{R})$ when $0<k<n$.

Proof: This proof combines the ingredients of the proofs of the last two corollaries. We need to show that

$$
\left\|\tau(t)\left(\varphi_{\varepsilon} f^{(k)}\right)-\left(\varphi_{\varepsilon} f^{(k)}\right)\right\|_{X} \longrightarrow 0
$$

as $t \rightarrow 0$, which we do by induction. Suppose that

$$
\left\|\tau(t)\left(\varphi_{\varepsilon} f^{(j)}\right)-\left(\varphi_{\varepsilon} f^{(j)}\right)\right\|_{X} \longrightarrow 0,
$$

when $0 \leqslant j<k$. Observe that

$$
\begin{aligned}
& \tau(t)\left(\varphi_{\varepsilon} f^{(k)}\right)-\left(\varphi_{\varepsilon} f^{(k)}\right) \\
& \quad=\tau(t)\left(\varphi_{\varepsilon} f\right)^{(k)}-\left(\varphi_{\varepsilon} f\right)^{(k)}-\sum_{j=0}^{k-1}\left(\begin{array}{l}
k \\
j
\end{array}\right)\left[\tau(t)\left(\varphi_{\varepsilon}^{(k-j)} f^{(j)}\right)-\left(\varphi_{\varepsilon}^{(k-j)} f^{(j)}\right)\right] .
\end{aligned}
$$

Now

$$
\begin{aligned}
& \left\|\tau(t)\left(\varphi_{\varepsilon} f\right)^{(k)}-\left(\varphi_{\varepsilon} f\right)^{(k)}\right\|_{X} \\
& \quad=\left\|\left(\tau(t)\left(\varphi_{\varepsilon} f\right)-\left(\varphi_{\varepsilon} f\right)\right)^{(k)}\right\|_{X} \\
& \quad \leqslant C_{X} C_{k, n}\left\|\tau(t)\left(\varphi_{\varepsilon} f\right)-\left(\varphi_{\varepsilon} f\right)\right\|_{X}^{1-k / n}\left\|\left(\tau(t)\left(\varphi_{\varepsilon} f\right)-\left(\varphi_{\varepsilon} f\right)\right)^{(n)}\right\|_{X}^{k / n} \\
& \quad \longrightarrow 0
\end{aligned}
$$

as $t \rightarrow 0$, since $\left\|\tau(t)\left(\varphi_{\varepsilon} f\right)-\left(\varphi_{\varepsilon} f\right)\right\|_{X} \rightarrow 0$ while $\left\|\left(\tau(t)\left(\varphi_{\varepsilon} f\right)-\left(\varphi_{\varepsilon} f\right)\right)^{(n)}\right\|_{X}$ is bounded as $t \rightarrow 0$, by the arguments of the previous corollaries. Further,

$$
\begin{aligned}
& \left\|\tau(t)\left(\varphi_{\varepsilon}^{(k-j)} f^{(j)}\right)-\left(\varphi_{\varepsilon}^{(k-j)} f^{(j)}\right)\right\|_{X} \\
& \leqslant\left\|\left(\tau(t) \varphi_{\varepsilon}^{(k-j)}-\varphi_{\varepsilon}^{(k-j)}\right) \tau(t) f^{(j)}\right\|_{X}+\left\|\varphi_{\varepsilon}^{(k-j)}\left(\tau(t) f^{(j)}-f^{(j)}\right)\right\|_{X} \\
& \leqslant \\
& \quad C_{X, m}\left\|\tau(t) \varphi_{\varepsilon}^{(k-j)}-\varphi_{\varepsilon}^{(k-j)}\right\|_{C^{m}}\left\|\tau(t) f^{(j)}\right\|_{X} \\
& \quad+C_{X, m}\left\|\varphi_{\varepsilon}^{(k-j)}\left(\varphi_{\varepsilon / 2}\right)^{-1}\right\|_{C^{m}}\left\|\varphi_{\varepsilon / 2}\left(\tau(t) f^{(j)}-f^{(j)}\right)\right\|_{X} \\
& \quad \longrightarrow 0
\end{aligned}
$$


as $t \rightarrow 0$, by straightforward estimates of $\varphi_{\varepsilon}$ and its derivatives, and the inductive hypothesis.

EXAmples. The Lorentz spaces $L^{p, q}(\mathbb{R})$ (where $1<p<\infty$ and $1 \leqslant q \leqslant \infty$ ) are dual spaces except when $q=1$, and are covered by the theorem. When $q=1$, they are covered by Corollary 4 .

The amalgams $\ell^{q}\left(L^{p}\right)$ are covered by the theorem if $p>1$. If $q<\infty$ and $X=$ $\ell^{q}(M)$, where $M$ denotes the space of bounded complex measures, then $X_{0}=\ell^{q}\left(L^{1}\right)$. If $X=\ell^{\infty}(M)$, then $X_{1}=\ell^{\infty}\left(L^{1}\right)$.

REMARKs. It should be noted that the constant obtained in the theorem is, in general, not best possible. For example, it is easy to show that the best constant when $X=L^{2}$ is 1 , by using the Fourier transform and Hölder's inequality. The point of the theorem is that there is a constant which works for all $D(\mathbb{R})$-full, $\tau$-stable, Banach spaces for which the translation constant $C_{X}$ of formula (1) is in a given range.

The hypothesis of Corollary 2 can be varied a little without changing the conclusion: more precisely, we may assume that $f$ is in $X(\mathbb{R})$ and its generalised derivative $f^{(n)}$ is in $X_{u}(\mathbb{R})$. Similarly, the hypothesis of Corollary 3 can also be varied.

If we are interested in proving additive inequalities, that is, those of the form

$$
\left\|f^{(k)}\right\|_{X} \leqslant C\left(\|f\|_{X}+\left\|f^{(n)}\right\|_{X}\right)
$$

then more can be said. Indeed, the condition that $X$ be $\tau$-stable can be replaced by the condition that translations act continuously on $X$ (that is, the map $(t, f) \mapsto \tau(t) f$ from $\mathbb{R} \times X$ to $X$ is continuous, which implies that $\|\tau(t) f\|_{X} \leqslant \Omega(t)\|f\|_{X}$ for all $f$ in $X$ and $t$ in $\mathbb{R}$, where $\Omega(t)$ grows at most exponentially as $|t|$ grows). By writing a function $f$ as $\psi * f+(f-\psi * f)$, where $\psi$ is a suitable test function, one sees that

$$
\left\|f^{(k)}\right\|_{X} \leqslant\left\|\psi^{(k)} * f\right\|_{X}+\left\|(f-\psi * f)^{(k)}\right\|_{X} .
$$

The first term on the right hand side can be controlled by a weighted $L^{1}$-norm of $\psi^{(k)}$ multiplied by $\|f\|_{X}$, and the second, after some integrations by parts, by a weighted $L^{1}$ norm of an $(n-k)$-fold integral of $\delta-\psi$ multiplied by $\left\|f^{(n)}\right\|_{X}$, where $\delta$ denotes the Dirac delta distribution. The conclusion at which one arrives is that the constant in the inequality (2) can be taken to depend only on $k$ and $n$ and the growth rate $\Omega(t)$. This result applies to spaces such as $L^{p}(\mathbf{R}, w)$, where the weight $w$ does not vanish or grow too fast; the weights $w(x)=(1+|x|)^{\alpha}$, where $\alpha$ is real, are examples of admissible weights.

\section{REFERENCES}

[1] H.H. Bang, 'A remark on the Kolmogorov-Stein inequality', J. Math. Anal. Appl. 203 (1996), 861-867.

[2] J.P. Bertrandias and C. Dupuis, 'Transformation de Fourier sur les espaces $I^{p}\left(L^{p^{\prime}}\right)$ ', $A n n$. Inst. Fourier Grenoble 29 (1979), 189-206. 
[3] W.R. Bloom, 'Estimates for the Fourier transform', Math. Scientist 10 (1985), 65-81.

[4] M.W. Certain and T.G. Kurtz, 'Landau-Kolmogorov inequalities for semigroups and groups', Proc. Amer. Math. Soc. 63 (1977), 226-230.

[5] J.J.F. Fournier, 'On the Hausdorff-Young theorem for amalgams', Monatsh. Math. 95 (1983), 117-135.

[6] F. Holland, 'Harmonic analysis on amalgams of $L^{p}$ and $l^{q}, J$. London Math. Soc. 10 (1975), 295-305.

[7] F. Holland, 'On the representation of functions as Fourier transforms of unbounded measures', Proc. London Math. Soc. 30 (1975), 347-365.

[8] A.N. Kolmogorov, 'On inequalities between upper bounds of the successive derivatives of an arbitrary function on an infinite interval', Uchen. Zap. Moskov. Gos. Uni. 30 (1939), 3-16.

[9] M.K. Kwong and A. Zettl, Norm inequalities for derivatives and differences, Lecture Notes in Math. 1536 (Springer-Verlag, Berlin, Heidelberg, New York, 1992).

[10] E.M. Stein, 'Functions of exponential type', Ann. Math. 65 (1957), 582-592.

[11] V.M. Tikhomirov and G.G. Magaril-Il'jaev, 'Inequalities for derivatives', in Kolmogorov, A.N., Selected Papers (Nauka, Moscow, 1985).

Institute of Mathematics

P.O. Box 61

10000 Bo Ho

Hanoi

Vietnam
Thainguyen Pedagogic College

Thainguyen

Vietnam 